\title{
Whole breast radiotherapy in prone and supine position: is there a place for multi-beam IMRT?
}

\author{
Thomas Mulliez ${ }^{1 * \dagger}$, Bruno Speleers ${ }^{2 \dagger}$, Indira Madani ${ }^{1}$, Werner De Gersem² ${ }^{2}$ Liv Veldeman ${ }^{1}$ and Wilfried De Neve
}

\begin{abstract}
Background: Early stage breast cancer patients are long-term survivors and finding techniques that may lower acute and late radiotherapy-induced toxicity is crucial. We compared dosimetry of wedged tangential fields (W-TF), tangential field intensity-modulated radiotherapy (TF-IMRT) and multi-beam IMRT (MB-IMRT) in prone and supine positions for whole-breast irradiation (WBI).

Methods: MB-IMRT, TF-IMRT and W-TF treatment plans in prone and supine positions were generated for 18 unselected breast cancer patients. The median prescription dose to the optimized planning target volume (PTV optim) was $50 \mathrm{~Gy}$ in 25 fractions. Dose-volume parameters and indices of conformity were calculated for the PTV optim and organs-at-risk.
\end{abstract}

Results: Prone MB-IMRT achieved $(p<0.01)$ the best dose homogeneity compared to WTF in the prone position and WTF and MB-IMRT in the supine position. Prone IMRT scored better for all dose indices. MB-IMRT lowered lung and heart dose $(p<0.05)$ in supine position, however the lowest ipsilateral lung doses $(p<0.001)$ were in prone position. In left-sided breast cancer patients population averages for heart sparing by radiation dose was better in prone position; though non-significant. For patients with a PTV optim volume $\geq 600 \mathrm{cc}$ heart dose was consistently lower in prone position; while for patients with smaller breasts heart dose metrics were comparable or worse compared to supine MB-IMRT. Doses to the contralateral breast were similar regardless of position or technique. Dosimetry of prone MB-IMRT and prone TF-IMRT differed slightly.

Conclusions: MB-IMRT is the treatment of choice in supine position. Prone IMRT is superior to any supine treatment for right-sided breast cancer patients and left-sided breast cancer patients with larger breasts by obtaining better conformity indices, target dose distribution and sparing of the organs-at-risk. The influence of treatment techniques in prone position is less pronounced; moreover dosimetric differences between TF-IMRT and MB-IMRT are rather small.

Keywords: Whole-breast irradiation, Prone position, Supine position, Wedged tangential fields, Intensity-modulated radiotherapy, Tangential field-IMRT, Multi-beam-IMRT

\section{Background}

Conventional radiotherapy (RT) using wedged tangential fields (W-TF) after breast-conserving surgery improves disease control and breast-cancer related survival. However prolonged follow-up showed an increased RT-induced risk of cardiac events and secondary lung and breast cancer in long-term survivors [1-3]. Therefore strategies for sparing

\footnotetext{
*Correspondence: thomas.mulliez@uzgent.be

${ }^{\dagger}$ Equal contributors

'Department of Radiotherapy, Ghent University Hospital, De Pintelaan 185, Ghent 9000, Belgium

Full list of author information is available at the end of the article
}

organs-at-risk (OARs), while maintaining an adequate dose coverage of the target are warranted.

In supine position the whole-breast clinical target volume $\left(\mathrm{CTV}_{\mathrm{WBI}}\right)$ is concave 1) enwrapping the lung and heart at the left side, and 2) medially adjoining the contralateral breast. Therefore parts of the ipsilateral lung, heart, and contralateral breast may receive intermediate to high doses with W-TF.

Intensity-modulated radiotherapy (IMRT) can provide advantages compared to W-TF. In supine position IMRT using a tangential two-beam set-up (TF-IMRT) can improve dose homogeneity; however its ability to reduce

\section{Biomed Central}


high-dose regions to the underlying heart and lung tissue appear to be limited [4,5]. Supine multi-beam IMRT (MB-IMRT) may overcome those limitations often at cost of low- or intermediate-dose spread over the contralateral breast and ipsilateral thoracic region [6-10].

Prone position modifies the target volume by gravity and moves the breast away from the chest wall. Prone W-TF has previously been used for large, pendulous breasts [11] to reduce fibrosis and improve cosmesis $[12,13]$. There are a few studies reporting improved dosimetry by prone TF-IMRT [14-16], though data on whole-breast MB-IMRT in prone position are lacking. Moreover, all dosimetric studies comparing prone and supine position used only non-multi-beam techniques [16-20]. We performed the present study to establish the effect of treatment technique (W-TF, TF-IMRT or MB-IMRT) and position (prone or supine) on dose coverage and heart and lung sparing.

\section{Methods}

Eighteen unselected early stage breast cancer patients 6 right-sided and 12 left-sided - presenting for wholebreast irradiation (WBI) without nodal irradiation after breast conserving surgery were included in this study. Three-mm thick computer-tomography scans were acquired with an Aquilion scanner (Toshiba Medical Systems, Tokyo, Japan) in all patients in prone and supine position. Patient set-up and delineation of the clinical and planning target volumes for WBI $\left(\mathrm{CTV}_{\mathrm{WBI}}\right.$ and PTV $_{\text {WBI }}$, respectively) and OARs in both treatment positions can be found elsewhere $[16,17]$. Extension of the PTV $V_{\text {WBI }}$ outside the skin into the air accounted for respiration-related breast movement or swelling of the breast during treatment. A flash region was created outside the patient's external contour by expanding the PTV $V_{\text {WBI }}$ with a $10 \mathrm{~mm}$ margin followed by subtraction of the patient's total scanned volume. This flash region was subsequently used in the optimization. A planning target volume for optimization $\left(\mathrm{PTV}_{\text {optim }}\right)$, a structure used during plan optimization, was generated by removing the in-air part and a $7 \mathrm{~mm}$-wide build-up region underneath the skin from the PTV $\mathrm{VWBI}_{\mathrm{WI}}$.

The dosimetric comparison was made for $6 \mathrm{MV}$ photon beams of an Elekta SLi18 linear accelerator (Elekta, Crawley, UK) equipped with a standard $1 \mathrm{~cm}$ leaf-width multileaf collimator (MLC). A median prescription dose to the $\mathrm{PTV}_{\text {optim }}$ was $50 \mathrm{~Gy}$ in 25 fractions of $2.0 \mathrm{~Gy}$ with the objective of $\geq 95 \%$ of the $\mathrm{PTV}_{\text {optim }}$ receiving $>95 \%$ of the prescribed dose and minimization of maximum dose, dose heterogeneity and "hot spots". In both positions TF-IMRT used the same gantry angles as W-TF with the collimator set at $0^{\circ}$ and the beams shaped around the PTV $V_{\text {WBI }}$ with the aid of the MLC. Figure 1 shows the 6-beam setup used in the MB-IMRT plans for rightsided breast tumors in supine position (a) and prone position (b). In both positions MB-IMRT used 6 coplanar beams shaped around the $\mathrm{PTV}_{\mathrm{WBI}}$ and as in TF-IMRT plans field-in-field segments were created avoiding the ipsilateral lung, heart (in case of left-sided breast tumors) and contralateral breast (for lateral beams in supine position, since medial beams did not traverse the contralateral breast).

A forward planning approach was used for the intensity-modulated and W-TF plans. The convolutionsuperposition dose engine of a Pinnacle version 9.0 treatment planning system (Philips Medical Systems, Andover, US) was used for dose computations between optimization cycles of intensity-modulated plans as well

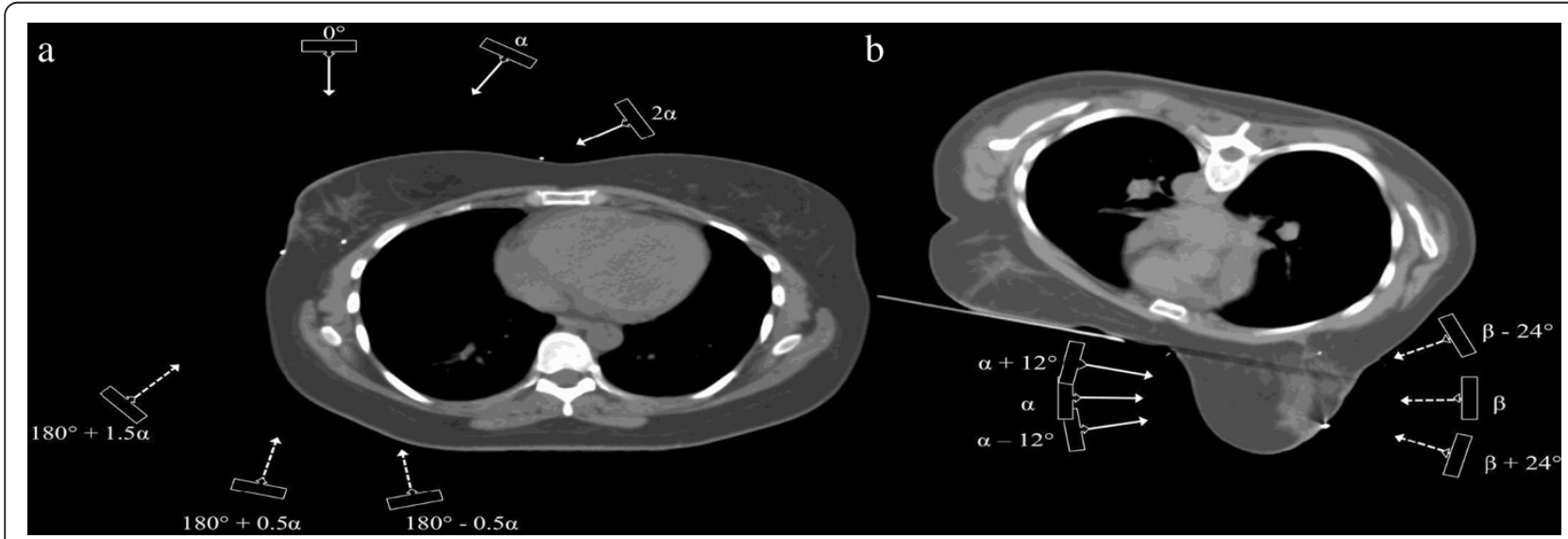

Figure 1 Multi-beam set-up in the prone and supine position. A 6-beam set-up used in the multi-beam intensity-modulated radiotherapy (MB-IMRT) plans for right-sided breast tumors in supine (a) and prone position (b). Gantry angles expressed in the Elekta coordinate system. The most inclined medial beam has the gantry angle of a tangential beam set by virtual simulation [21]. The gantry angles are $0^{\circ},|a|,|2 a|$, $180^{\circ}-0.5|\mathrm{a}|, 180^{\circ}+0.5|\mathrm{a}|$, and $180^{\circ}+1.5|\mathrm{a}|$ for supine MB-IMRT. The lateral gantry angles in prone MB-IMRT are $|\mathrm{a}|,|\mathrm{a}|+/-24^{\circ}$, the medial gantry angles are $|\beta|,|\beta|+/-12^{\circ}$. 
as for final plans. Monitor units and MLC shapes were optimized using the optimization tools described before [21]. During optimization, two patient geometries were taken into account: 1) dose computation for PTV $\mathrm{WWB}_{\mathrm{WBI}}$ was performed using a density override $\left(1 \mathrm{~g} / \mathrm{cm}^{3}\right)$ to the above-mentioned flash region; 2) dose computation for the PTV $V_{\text {WBI }}$ without build-up and OARs was performed without density overrides. To be able to compute both dose distributions in parallel, the patient data at the Pinnacle treatment planning system were duplicated: for the first patient dataset the flash region was set waterequivalent, while for the second patient dataset, the flash region remained at the density of the $\mathrm{CT}$ data (in essence, air outside the patient outline). To avoid hot spots outside regions of interest, a "matroska" sequence of shell structures [22] was generated outside the PTV ${ }_{\mathrm{WB}}$, which were taken into account during optimization. Dose computation for these shell structures was performed using the above-mentioned density override in the flash region. Also the dose update mechanism for changes in leaf positions during optimization took both patient geometries into account. This method was used mainly to account for substantial deformations of the breast during the course of treatments.

$D_{2}$ and $D_{98}$, or the dose exceeding $2 \%$ and $98 \%$ of the dose-volume histogram $(\mathrm{DVH})$ points, respectively, were used as surrogates for maximum and minimum dose. These were evaluated for the $\mathrm{PTV}_{\text {optim }}$, as well as dose homogeneity $\left(1-\left(D_{2}-D_{98} /\right.\right.$ median dose $\left.)\right)$. For the heart and ipsilateral lung $D_{2}$, mean dose $\left(D_{\text {mean }}\right), V_{5}, V_{10}, V_{20}$ and $\mathrm{V}_{25}$ or the proportion of the volume receiving at least 5 Gy, $10 \mathrm{~Gy}, 20 \mathrm{~Gy}$ and 25Gy, respectively, were extracted from the DVH data. For the contralateral breast $\mathrm{D}_{2}$ and $\mathrm{D}_{\text {mean }}$ were evaluated.

The following indices were also calculated for the PTV optim:

$$
\text { Jaccard index }=A \cap B / A \cup B
$$

Where $\mathrm{A}$ is the volume covered by the $\mathrm{PTV}_{\text {optim }}$ and $B$ is the volume covered by the $95 \%$ isodose, i.e., the volume receiving 47.5 Gy or more. The Jaccard index increases with increase in similarity or overlap between the target volume and the $95 \%$ isodose and is a measure of dose conformity of the treatment plan.

$$
\text { Dose-coverage index }=\mathrm{A} \cap \mathrm{B} 1 / \mathrm{A}
$$

Where B1 is the volume covered by the $95-107 \%$ isodose, i.e. the volume receiving between $47.5 \mathrm{~Gy}$ and 53.5 Gy. The dose-coverage index calculates the proportion of the target, in which the treatment-planning objectives for the target are met.

$$
\text { Mismatch index }=\mathrm{B} 2 / \mathrm{B}
$$

Where B2 is the volume covered by the $95 \%$ isodose and lying outside the $\mathrm{PTV}_{\text {optim. }}$. It is the fraction of the 95\% isodose non-overlapping the target. If the mismatch index is large, large amounts of normal tissues receive $95 \%$ of the prescription dose, i.e., $47.5 \mathrm{~Gy}$.

One-way analysis of variance (ANOVA) was used for a pairwise comparison of dose-volume parameters and indices between MB-IMRT, TF-IMRT and W-TF in the 2 treatment positions.

\section{Results}

One hundred-and-eight plans were generated. Figure 2 illustrates typical dose distributions obtained with the 3 techniques in prone and supine position.

\section{Dose homogeneity and dose coverage of the target}

Table 1 provides numerical data on target coverage and target dose distribution obtained with the 3 techniques in the prone and supine position. $\mathrm{D}_{2}$ is lowered in prone position resulting in improved dose homogeneity since $\mathrm{D}_{98}$ was similar for both positions. Significance was obtained for prone MB-IMRT versus all supine techniques and a trend $(\mathrm{p}=0.05)$ for prone TF-IMRT compared to supine $\mathrm{W}$-TF regarding $\mathrm{D}_{2}$; moreover prone MB-IMRT obtained better $(\mathrm{p}<0.01)$ dose homogeneity compared to supine $\mathrm{W}-\mathrm{TF}$ and MB-IMRT. Intensitymodulated techniques were able to improve dose homogeneity compared to conventional techniques in both positions, though significance $(\mathrm{p}=0.002)$ was only gained for prone MB-IMRT versus prone W-TF.

Prone WBI scored better for Jaccard and mismatch indices (Table 1). Prone MB-IMRT achieved better results than any supine treatment technique $(\mathrm{p} \leq 0.03$, both indices); followed by prone TF-IMRT versus supine TFIMRT and W-TF ( $\mathrm{p} \leq 0.001$, both indices). In supine position MB-IMRT $(\mathrm{p}<0.001)$ was the best and W-TF $(\mathrm{p}<0.001)$ was the worst technique for both indices. Prone IMRT improved significantly $(\mathrm{p}<0.01)$ dose coverage index: prone TF-IMRT vs. supine MB-IMRT and prone MB-IMRT vs. supine MB-IMRT and TF-IMRT.

\section{Dose-volume parameters in OARs}

Figure 3 illustrates cumulative DVHs of the ipsilateral lung (all patients) and heart (only left-sided patients), numerical data are presented in Table 2. Sparing $(p<0.001)$ of the ipsilateral lung by radiation dose was always superior in prone. There was little difference in ipsilateral lung dose between the 3 techniques in prone position, although $V_{10}$ and $V_{20}$ were significantly lower in prone MB-IMRT vs. prone $\mathrm{W}-\mathrm{TF}$. In supine position treatment technique did alter lung dose $(\mathrm{p}<0.05)$, MBIMRT achieved the best and W-TF the worst lung 


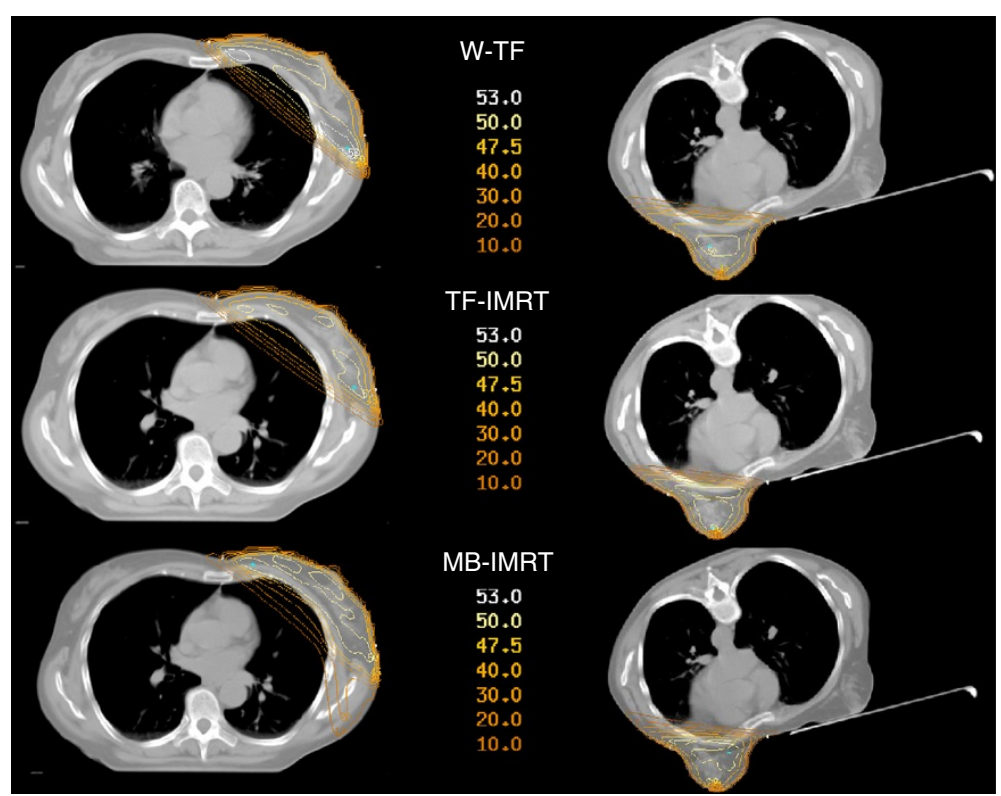

Figure 2 Isodose distributions (in Gy) of the 6 treatment plans for a left-sided patient in a transverse plane. Abbreviations: W-TF = wedged tangential fields; TF-IMRT = tangential field intensity-modulated radiotherapy; MB-IMRT = multi-beam intensity-modulated radiotherapy.

avoidance by radiation dose. A remarking feature is the modified ( $\mathrm{p}=0.003)$ ipsilateral lung volume in both positions. Mean \pm standard deviation for ipsilateral lung volume is $1504 \pm 401 \mathrm{cc}$ for prone position versus $1409 \pm$ 431cc for supine position.

Heart dose was lowered with MB-IMRT compared to TF-IMRT $\left(\mathrm{D}_{2}, \mathrm{D}_{\text {mean }}, \mathrm{V}_{5} ; \mathrm{p}=0.07,0.05\right.$ and 0.03 , respectively) and W-TF $\left(\mathrm{D}_{2}, \mathrm{~V}_{5}, \mathrm{p}=0.009\right.$ and 0.07 , respectively) in supine position. While in prone position the effect of treatment technique on heart dose is less pronounced. Population averages for heart dose metrics were non- significantly lowered in prone compared to supine position. Better heart sparing by radiation dose was consistently obtained in prone position for patients with a $\mathrm{PTV}_{\text {optim }}$ volume $\geq 600 \mathrm{cc}$. While for patients with a $\mathrm{PTV}_{\text {optim }}$ volume $<600 \mathrm{cc}$ heart dose metrics were comparable ( $2 / 5$ patients) or worse (3/5 patients) in prone position compared to supine MB-IMRT.

Neither treatment technique, nor set-up significantly changed doses in the contralateral breast, all procedures achieved a maximum dose $<5 \mathrm{~Gy}$ and mean dose $<1.5 \mathrm{~Gy}$ for all patients.

Table 1 Dose-volume parameters (a) and conformity indices (b) for the optimized planning target volume (PTV optim)

\begin{tabular}{|c|c|c|c|c|c|c|c|c|c|c|c|c|c|c|c|c|c|c|}
\hline & \multicolumn{6}{|c|}{$D_{2}[G y]$} & \multicolumn{6}{|c|}{$D_{98}[G y]$} & \multicolumn{6}{|c|}{ Dose homogeneity [\%] } \\
\hline & \multicolumn{3}{|c|}{ Prone } & \multicolumn{3}{|c|}{ Supine } & \multicolumn{3}{|c|}{ Prone } & \multicolumn{3}{|c|}{ Supine } & \multicolumn{3}{|c|}{ Prone } & \multicolumn{3}{|c|}{ Supine } \\
\hline & mean & SEM & SD & mean & SEM & SD & mean & SEM & SD & mean & SEM & SD & mean & SEM & SD & Mean & SEM & SD \\
\hline \multicolumn{19}{|l|}{ (a) } \\
\hline W-TF & 52.3 & 0.1 & 0.6 & 53.1 & 0.2 & 0.9 & 47.6 & $<0.1$ & 0.1 & 47.9 & $<0.1$ & 0.4 & 90.6 & 0.3 & 1.1 & 89.7 & 0.5 & 2.1 \\
\hline TF-IMRT & 52.0 & 0.2 & 0.8 & 52.6 & 0.2 & 0.8 & 47.8 & $<0.1$ & 0.3 & 47.9 & 0.1 & 0.5 & 91.8 & 0.4 & 1.7 & 90.7 & 0.5 & 2.3 \\
\hline \multirow[t]{4}{*}{ MB-IMRT } & 51.6 & 0.2 & 0.7 & 52.6 & 0.1 & 0.6 & 47.9 & $<0.1$ & 0.2 & 47.7 & $<0.1$ & 0.2 & 92.5 & 0.3 & 1.4 & 90.3 & 0.3 & 1.2 \\
\hline & \multicolumn{6}{|c|}{ Jaccard index [\%] } & \multicolumn{6}{|c|}{ Dose-coverage index [\%] } & \multicolumn{6}{|c|}{ Mismatch index [\%] } \\
\hline & \multicolumn{3}{|c|}{ Prone } & \multicolumn{3}{|c|}{ Supine } & \multicolumn{3}{|c|}{ Prone } & \multicolumn{3}{|c|}{ Supine } & \multicolumn{3}{|c|}{ Prone } & \multicolumn{3}{|c|}{ Supine } \\
\hline & mean & SEM & SD & mean & SEM & SD & mean & SEM & SD & mean & SEM & SD & mean & SEM & SD & mean & SEM & SD \\
\hline (b) & & & & & & & & & & & & & & & & & & \\
\hline W-TF & 74.9 & 2.0 & 8.5 & 52.9 & 3.6 & 15.2 & 97.2 & 0.2 & 1.0 & 96.2 & 0.6 & 2.4 & 23.9 & 2.0 & 8.6 & 46.8 & 3.6 & 15.4 \\
\hline TF-IMRT & 74.8 & 1.5 & 6.2 & 64.6 & 2.1 & 8.9 & 97.7 & 0.2 & 0.8 & 96.6 & 0.3 & 1.2 & 24.4 & 1.5 & 6.4 & 34.7 & 2.1 & 9.1 \\
\hline MB-IMRT & 77.1 & 1.4 & 5.9 & 70.5 & 1.6 & 6.7 & 97.8 & 0.1 & 0.6 & 96.5 & 0.2 & 1.0 & 22.1 & 1.4 & 6.0 & 28.5 & 1.6 & 6.8 \\
\hline
\end{tabular}

Abbreviations: SEM Standard error of the mean, SD Standard deviation, W-TF Wedged tangential fields, TF-IMRT Tangential field intensity-modulated radiation therapy, MB-IMRT Multi-beam intensity-modulated radiation therapy. 


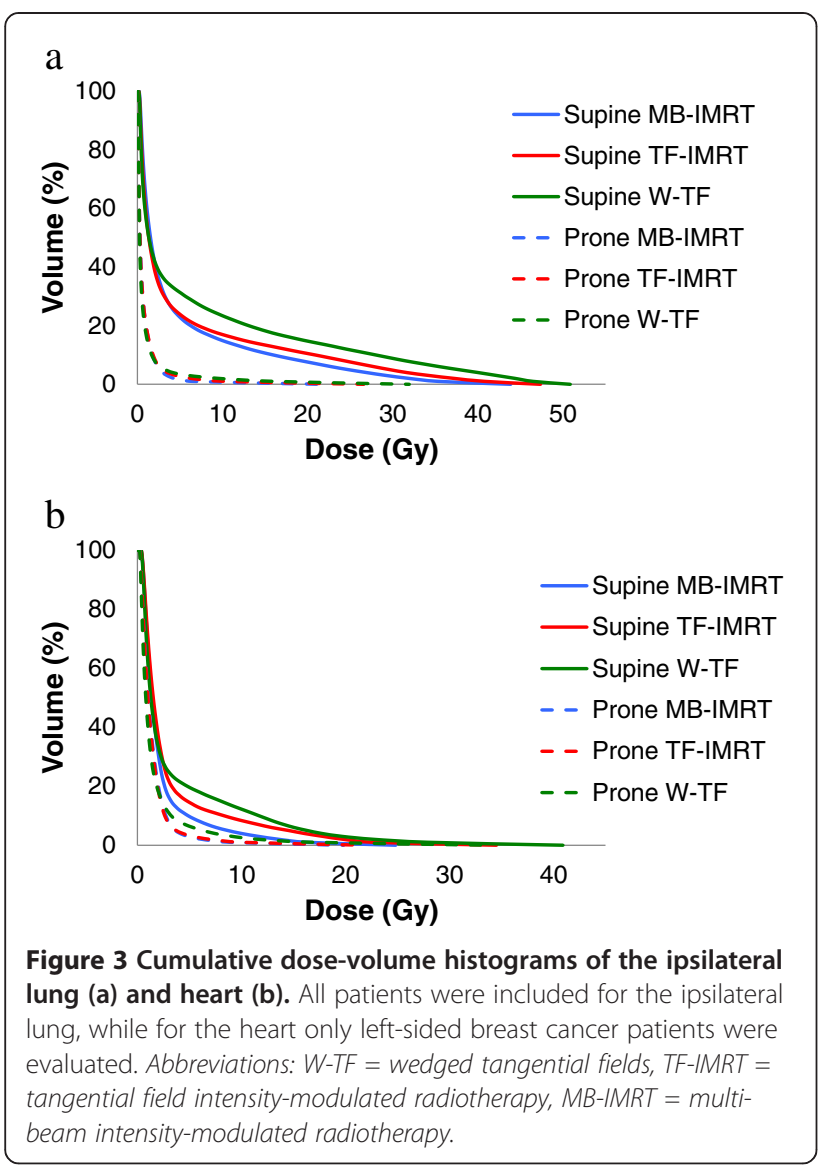

\section{Discussion}

In supine position IMRT techniques obtain a higher Jaccard index, i.e. superior dose conformity, and less mismatch compared to W-TF with MB-IMRT being the superior technique for both indices. Dose conformity, coverage and mismatch are even better for the prone techniques, becoming statistically significant in prone IMRT plans. This is not surprising, since prone position results in a less concave breast volume. Therefore dose to the axillary and shoulder region is substantially reduced and less of the prescription dose can be expected to be out of the target. Our results confirm the reduction of dose inhomogeneity, with IMRT-techniques compared to standard W-TF. Though differences were rather small and non-significant in supine position, which could be explained by the use of non-mixture beam energies. Prone as compared to supine IMRT does improve dose homogeneity and hot spots with the best results in prone MB-IMRT plans. Our results are in agreement with other publications on prone IMRT. Goodman et al. [15] demonstrated a maximum dose in the target exceeding $110 \%$ with prone W-TF in 16 of 20 patients as compared to 1 patient with prone IMRT (TF-IMRT). Another study comparing MB-IMRT, TF-IMRT and 3D-CRT treatment plans of 5 patients planned in prone position reported significantly higher dose homogeneity of MB-IMRT plans vs. TF-IMRT $(\mathrm{p}=0.003)$ and 3D-CRT plans $(\mathrm{p}=0.03)$ [23]. Hardee et al. [14] observed a maximum dose reduction and improved median dose homogeneity in a prone TF-IMRT vs. 3DCRT patient cohort. Moreover a $9 \%$-decrease of grade 2 dermatitis and a $16 \%$-reduction of grade $\geq 2$ hyperpigmentation were found in the IMRT group. We expect that improved dose homogeneity and hot spots achieved by prone IMRT - either MB-IMRT or TF-IMRT - will yield lower skin toxicity and better cosmesis $[4,5,24]$.

Lung irradiation was lowered with the MB-IMRT technique in supine position, though sparing of the ipsilateral lung appeared to be depending more on the treatment position than on the treatment technique. Prone position resulted in a spectacular decrease in lung dose, which is in coherence with other data [16-20]. The decrease in lung dose in prone position might also be attributed by the $7 \%$ increase in ipsilateral lung volume, for which we don't have an explanation. All prone treatment techniques showed similar lung dose metrics.

Left-sided breast cancer patients are at risk of radiation-induced cardiac events [2], emphasizing the importance of using more sophisticated techniques to lower the heart dose. In supine position, MB-IMRT is able to lower the heart dose compared to the other techniques as shown both in our data and in other publications [7-9]. In prone position different treatment techniques have less effect on heart dose, especially between IMRT-techniques. Even with MB-IMRT, only the minority of patients $(3 / 12)$ benefitted from supine position; which is in coherence with other data $[18,20]$.

Table 2 Mean \pm standard deviation for ipsilateral lung (all patients) and heart (only left-sided patients) dose metrics

\begin{tabular}{|c|c|c|c|c|c|c|c|c|c|c|c|c|}
\hline \multirow[t]{3}{*}{ Technique } & \multicolumn{6}{|c|}{ Ipsilateral lung } & \multicolumn{6}{|c|}{ Heart } \\
\hline & \multicolumn{2}{|c|}{$D_{\text {mean }}[G y]$} & \multicolumn{2}{|c|}{$\mathrm{V}_{20}[\%]$} & \multicolumn{2}{|c|}{$\mathrm{V}_{25}[\%]$} & \multicolumn{2}{|c|}{$D_{\text {mean }}[G y]$} & \multicolumn{2}{|c|}{$\mathrm{v}_{20}[\%]$} & \multicolumn{2}{|c|}{$\mathrm{V}_{25}[\%]$} \\
\hline & Prone & Supine & Prone & Supine & Prone & Supine & Prone & Supine & Prone & Supine & Prone & Supine \\
\hline W-TF & $1.2 \pm 0.6$ & $7.7 \pm 4.5$ & $0.9 \pm 1.0$ & $13.5 \pm 10.2$ & $0.7 \pm 0.8$ & $12.1 \pm 9.3$ & $1.9 \pm 1.1$ & $3.9 \pm 3.4$ & $1.2 \pm 0.6$ & $4.9 \pm 1.9$ & $0.8 \pm 1.7$ & $4.0 \pm 5.8$ \\
\hline TF-IMRT & $1.1 \pm 0.5$ & $5.7 \pm 3.1$ & $0.5 \pm 0.7$ & $9.8 \pm 7.0$ & $0.3 \pm 0.5$ & $8.5 \pm 6.4$ & $1.6 \pm 0.5$ & $3.3 \pm 2.5$ & $0.4 \pm 0.2$ & $3.4 \pm 1.4$ & $0.3 \pm 0.6$ & $2.9 \pm 4.3$ \\
\hline MB-IMRT & $0.9 \pm 0.4$ & $5.1 \pm 2.6$ & $0.2 \pm 0.4$ & $7.6 \pm 6.2$ & $0.1 \pm 0.3$ & $6.4 \pm 5.5$ & $1.6 \pm 0.4$ & $2.5 \pm 1.7$ & $0.3 \pm 0.1$ & $1.9 \pm 0.9$ & $0.2 \pm 0.3$ & $1.4 \pm 2.2$ \\
\hline
\end{tabular}

Abbreviations: $D_{\text {mean }}$ Mean dose, $V_{20}$ and $V_{25}$ Partial volume receiving at least 20 Gy and 25 Gy, respectively, W-TF Wedged tangential fields, TF-IMRT Tangential field intensity-modulated radiation therapy, MB-IMRT Multi-beam intensity-modulated radiation therapy. 
Moreover consistent better heart dose metrics were achieved in prone position for patients with a $\mathrm{PTV}_{\text {optim }}$ volume of $\geq 600 \mathrm{cc}$. A limitation of this study is the absence of dose parameters of the left descending coronary artery, since this is likely associated with increased cardiac mortality.

The introduction of supine MB-IMRT was not successful because of its complexity, increase in dose to the contralateral breast and higher integral dose [7-9]. In contrast with these studies we selected beams that avoided the contralateral breast and removed beams that included too much lung tissue. In this way reducing the dose in the ipsilateral lung with MB-IMRT, both in supine and prone position, was not at cost of low-dose spread over the lung or heart as illustrated by the DVHs (Figure 3). The dose to the contralateral breast was not increased with MB-IMRT either, moreover a maximum dose $<5 \mathrm{~Gy}$ and mean dose $<1.5 \mathrm{~Gy}$ was obtained for all patients.

As a consequence of the reduced ipsilateral lung and heart dose, better dose distribution and dose coverage, prone IMRT is superior to any supine technique for leftsided patients with larger breasts $\left(\mathrm{PTV}_{\text {optim }} \geq 600 \mathrm{cc}\right)$ and all right-sided patients. While for left-sided patients with smaller breasts individual comparative planning should be made between supine MB-IMRT and prone IMRT in order to choose the best technique for clinical execution. The dosimetric differences between prone TF-IMRT and prone MB-IMRT are rather small. Whether these "small" dosimetric benefits would cause a clinical benefit is unknown. The more complex and time consuming planning procedure and beam delivery of prone MB-IMRT should also be considered.

\section{Conclusions}

MB-IMRT is the preferred technique in supine position by providing better coverage indices of the target and sparing of organs-at-risk. However, prone IMRT is superior to any supine technique for right-sided breast cancer patients and left-sided breast cancer patients with larger breasts. The impact of treatment techniques in prone position is less prominent; moreover dosimetric differences between both IMRT-techniques are rather small.

\section{Abbreviations}

RT: Radiotherapy; WBI: Whole breast irradiation; OARs: Organs-at-risk W-TF: Wedged tangential fields; IMRT: Intensity-modulated radiotherapy; TF-IMRT: Tangential field intensity-modulated radiotherapy; MB-IMRT: Multibeam intensity-modulated radiotherapy; $C T V_{W B}$ : Whole-breast clinical target volume; PTV WBi $_{\text {: }}$ Whole-breast planning target volume; PTV optim: $_{\text {Planning }}$ target volume for optimization; DVH: Dose-volume histogram; $D_{\text {mean }}$ : Mean dose; $D_{2}$ and $D_{98}$ : Dose exceeding $2 \%$ and $98 \%$ of the DVH points, respectively; $V_{5}, V_{10}, V_{20}$ and $V_{25}$ : Partial volume receiving at least $5 \mathrm{~Gy}$, $10 \mathrm{~Gy}, 20 \mathrm{~Gy}$ and $25 \mathrm{~Gy}$, respectively; ANOVA: Analysis of variance.
}

\section{Competing interests}

The authors declare that they have no competing interests.

\section{Authors' contributions}

IM, WDG, LV and WDN participated in the design and coordination of the study and helped to draft the manuscript. TM and BS conceived of the study, participated in the design and coordination of the study, participated in the treatment planning, carried out the dose calculations, performed the statistical analysis and drafted the manuscript. All authors read and approved the final manuscript.

\section{Author details}

1Department of Radiotherapy, Ghent University Hospital, De Pintelaan 185, Ghent 9000, Belgium. ${ }^{2}$ Ghent University, De Pintelaan 185, Ghent 9000, Belgium.

Received: 28 January 2013 Accepted: 18 June 2013

Published: 24 June 2013

\section{References}

1. Henson KE, McGale P, Taylor C, Darby SC: Radiation-related mortality from heart disease and lung cancer more than 20 years after radiotherapy for breast cancer. Br J Cancer 2013, 108:179-182.

2. Clarke M, Collins R, Darby S, et al: Effects of radiotherapy and of differences in the extent of surgery for early breast cancer on local recurrence and 15-year survival: an overview of the randomised trials. Lancet 2005, 366:2087-2106.

3. Early Breast Cancer Trialists' Collaborative G, Darby S, McGale P, et al: Effect of radiotherapy after breast-conserving surgery on 10-year recurrence and 15-year breast cancer death: meta-analysis of individual patient data for 10,801 women in 17 randomised trials. Lancet 2011, 378:1707-1716.

4. Barnett GC, Wilkinson JS, Moody AM, et al: Randomized controlled trial of forward-planned intensity modulated radiotherapy for early breast cancer: interim results at 2 years. Int J Radiat Oncol Biol Phys 2012, 82:715-723.

5. Veldeman L, Madani I, Hulstaert F, De Meerleer G, Mareel M, De Neve W: Evidence behind use of intensity-modulated radiotherapy: a systematic review of comparative clinical studies. Lancet Oncology 2008, 9:367-375.

6. Borca VC, Franco P, Catuzzo P, et al: Does TomoDirect 3DCRT represent a suitable option for post-operative whole breast irradiation? A hypothesis-generating pilot study. Radiat Oncol 2012, 7:211.

7. Coon AB, Dickler A, Kirk MC, et al: Tomotherapy and Multifield IntensityModulated Radiotherapy Planning Reduce Cardiac Doses in Left-Sided Breast Cancer Patients with Unfavorable Cardiac Anatomy. Int J Radiat Oncol Biol Phys 2010, 72:104-110.

8. Beckham WA, Popescu CC, Patenaude W, Wai ES, Olivotto IA: Is multibeam IMRT better than standard treatment for patients with left-sided breast cancer? Int J Radiat Oncol Biol Phys 2007, 69:918-924.

9. Fogliata A, Clivio A, Nicolini G, Vanetti E, Cozzi L: A treatment planning study using non-coplanar static fields and coplanar arcs for whole breast radiotherapy of patients with concave geometry. Radiother Oncol 2007, 85:346-354.

10. Rudat V, Alaradi AA, Mohamed A, Ai-Yahya K, Altuwaiji S: Tangential beam IMRT versus tangential beam 3D-CRT of the chest wall in postmastectomy breast cancer patients: a dosimetric comparison. Radiat Oncol 2011, 6:26.

11. Merchant TE, McCormick B: Prone position breast irradiation. Int J Radiat Oncol Biol Phys 1994, 30:197-203.

12. Gray JR, McCormick B, Cox L, Yahalom J: Primary breast irradiation in large-breasted or heavy women: analysis of cosmetic outcome. Int J Radiat Oncol Biol Phys 1991, 21:347-354.

13. Grann A, McCormick B, Chabner ES, et al: Prone breast radiotherapy in early-stage breast cancer: a preliminary analysis. Int J Radiat Oncol Biol Phys 2000, 47:319-325

14. Hardee ME, Raza S, Becker SJ, et al: Prone hypofractionated whole-breast radiotherapy without a boost to the tumor bed: comparable toxicity of IMRT versus a 3D conformal technique. Int J Radiat Oncol Biol Phys 2012, 82:e415-423.

15. Goodman KA, Hong L, Wagman R, Hunt MA, McCormick B: Dosimetric analysis of a simplified intensity modulation technique for prone breast radiotherapy. Int J Radiat Oncol Biol Phys 2004, 60:95-102.

16. Veldeman L, Speleers B, Bakker $M$, et al: Preliminary results on setup precision of prone-lateral patient positioning for whole breast irradiation. Int J Radiat Oncol Biol Phys 2010, 78:111-118. 
17. Veldeman L, De Gersem W, Speleers B, et al: Alternated Prone and Supine Whole-Breast Irradiation Using IMRT: Setup Precision, Respiratory Movement and Treatment Time. Int J Radiat Oncol Biol Phys 2012, 82:2055-2064.

18. Kirby AM, Evans PM, Donovan EM, Convery HM, Haviland JS, Yarnold JR: Prone versus supine positioning for whole and partial-breast radiotherapy: a comparison of non-target tissue dosimetry. Radiother Oncol 2010, 96:178-184.

19. Varga Z, Hideghety K, Mezo T, Nikolenyi A, Thurzo L, Kahan Z: Individual positioning: a comparative study of adjuvant breast radiotherapy in the prone versus supine position. Int I Radiat Oncol Biol Phys 2009, 75:94-100

20. Lymberis SC, Dewyngaert JK, Parhar P, et al: Prospective Assessment of Optimal Individual Position (Prone Versus Supine) for Breast Radiotherapy: Volumetric and Dosimetric Correlations in 100 Patients. Int J Radiat Oncol Biol Phys 2012, 84:902-909.

21. Van Vaerenbergh K, De Gersem W, Vakaet L, et al: Automatic generation of a plan optimization volume for tangential field breast cancer radiation therapy. Strahlenther Onkol 2005, 181:82-8.

22. De Neve W, Wu Y, Ezzel G: Practical IMRT planning. In Image-guided IMRT. Edited by Bortfeld T, Schmidt-Ulrich R, De Neve W, Wazer D. Berlin, Heidelberg: Springer; 2006:47-59.

23. Ahunbay EE, Chen GP, Thatcher S, et al: Direct aperture optimizationbased intensity-modulated radiotherapy for whole breast irradiation. Int J Radiat Oncol Biol Phys 2007, 67:1248-1258.

24. Harsolia A, Kestin L, Grills I, et al: Intensity-modulated radiotherapy results in significant decrease in clinical toxicities compared with conventional wedge-based breast radiotherapy. Int J Radiat Oncol Biol Phys 2007, 68:1375-1380

doi:10.1186/1748-717X-8-15

Cite this article as: Mulliez et al:: Whole breast radiotherapy in prone and supine position: is there a place for multi-beam IMRT?. Radiation Oncology 2013 8:151.

\section{Submit your next manuscript to BioMed Central and take full advantage of:}

- Convenient online submission

- Thorough peer review

- No space constraints or color figure charges

- Immediate publication on acceptance

- Inclusion in PubMed, CAS, Scopus and Google Scholar

- Research which is freely available for redistribution 